\title{
Modified Piyavskii's Global One-Dimensional Optimization of a Differentiable Function
}

\author{
Rachid Ellaia, Mohamed Zeriab Es-Sadek", Hasnaa Kasbioui \\ Laboratory of Study and Research in Applied Mathematics (LRMA), Mohammadia School of Engineering, \\ Mohammed V University (Adgal), Av Ibn Sina, Rabat, Morocco \\ Email: "essadekzeriab@yahoo.fr
}

Received June 21, 2012; revised August 28, 2012; accepted September 4, 2012

\begin{abstract}
Piyavskii's algorithm maximizes a univariate function satisfying a Lipschitz condition. We propose a modified Piyavskii's sequential algorithm which maximizes a univariate differentiable function $f$ by iteratively constructing an upper bounding piece-wise concave function $\Phi$ of $f$ and evaluating $f$ at a point where $\Phi$ reaches its maximum. We compare the numbers of iterations needed by the modified Piyavskii's algorithm $\left(n_{C}\right)$ to obtain a bounding piece-wise concave function $\Phi$ whose maximum is within $\varepsilon$ of the globally optimal value $f_{\text {opt }}$ with that required by the reference sequential algorithm $\left(n_{r e f}\right)$. The main result is that $n_{C} \leq 2 n_{r e f}+1$ and this bound is sharp. We also show that the number of iterations needed by modified Piyavskii's algorithm to obtain a globally $\varepsilon$-optimal value together with a corresponding point $\left(n_{B}\right)$ satisfies $n_{B} \leq 4 n_{r e f}+1$ Lower and upper bounds for $n_{r e f}$ are obtained as functions of $f(x), \varepsilon, M_{1}$ and $M_{0}$ where $M_{0}$ is a constant defined by $M_{0}=\sup _{x \in[a, b]}-f^{\prime \prime}(x)$ and $M_{1} \geq M_{0}$ is an evaluation of $M_{0}$.
\end{abstract}

Keywords: Global Optimization; Piyavskii's Algorithm

\section{Introduction}

We consider the following general global optimization problems for a function defined on a compact set

$$
X \subseteq R \quad-0.9 e x 0.15 e x 1.4 e x \quad 0.9 e x^{m}:
$$

$(P)$ Find

$$
\left(x^{*}, f^{*}\right) \in X \times R-0.9 e x 0.15 \operatorname{ex} 1.4 \operatorname{ex} 0.9 e x
$$

such that

$$
f^{*}=f\left(x^{*}\right) \geq f(x) \quad \forall x \in X .
$$

$\left(P_{1}\right)$ Find $x_{\text {opt }} \in X$ such that $f_{\text {opt }}=f\left(x_{\text {opt }}\right) \geq f^{*}-\varepsilon$, where $\varepsilon$ is a small positive constant.

Many recent papers and books propose several approaches for the numerical resolution of the problem $(P)$ and give a classification of the problems and their methods of resolution. For instance, the book of Horst and Tuy [1] provides a general discussion concerning deterministic algorithms. Piyavskii [2,3] proposes a deterministic sequential method which solves $(P)$ by iteratively constructing an upper bounding function $F$ of $f$ and evaluating $f$ at a point where $F$ reaches its maximum, Shubert [4], Basso [5,6], Schoen [7], Shen and Zhu [8] and Horst and Tuy [9] give a special aspect of its application by examples involving functions satisfying a Lipschitz condition and propose other formulations of the

"Corresponding author.
Piyavskii's algorithm, Sergeyev [10] use a smooth auxiliary functions for an upper bounding function, Jones $\mathrm{et} \mathrm{al}$. [11] consider a global optimization without the Lipschitz constant. Multidimensional extensions are proposed by Danilin and Piyavskii [12], Mayne and Polak [13], Mladineo [14] and Meewella and Mayne [15], Evtushenko [16], Galperin [17] and Hansen, Jaumard and Lu $[18,19]$ propose other algorithms for the problem $(P)$ or its multidimensional case extension. Hansen and Jaumard [20] summarize and discuss the algorithms proposed in the literature and present them in a simplified and uniform way in a high-level computer language. Another aspect of the application of Piyavskii's algorithm has been developed by Brent [21], the requirement is that the function is defined on a compact interval, with a bounded second derivative. Jacobsen and Torabi [22] assume that the differentiable function is the sum of a convex and concave functions. Recently, a multivariate extension is proposed by Breiman and Cutler [23] which use the Taylor development of $f$ to build an upper bounding function of $f$. Baritompa and Cutler [24] give a variation and an acceleration of the Breiman and Cutler's method. In this paper, we suggest a modified Piyavskii's sequential algorithm which maximizes a univariate differentiable function $f$. The theoretical study of the number of iterations of Piyavskii's algorithm was initiated by Dani- 
lin [25]. Danilin's result was improved by Hansen, Jaumard and $\mathrm{Lu} \mathrm{[26].} \mathrm{In} \mathrm{the} \mathrm{same} \mathrm{way,} \mathrm{we} \mathrm{develop} \mathrm{a} \mathrm{refer-}$ ence algorithm in order to study the relationship between $n_{B}$ and $n_{\text {ref }}$ where $n_{B}$ denotes the number of iterations used by the modified Piyavskii's algorithm to obtain an $\varepsilon$-optimal value, and $n_{\text {ref }}$ denotes the number of iterations used by a reference algorithm to find an upper bounding function, whose maximum is within $\varepsilon$ of the maximum of $f$. Our main result is that $n_{B} \leq 4 n_{\text {ref }}+1$. The last purpose of this paper is to derive bounds for $n_{r e f}$. Lower and upper bounds for $n_{r e f}$ are obtained as functions of $f(x), \varepsilon, M_{1}$ and $M_{0}$ where $M_{0}$ is a constant defined by

$$
M_{0}=\sup _{x \in[a, b]}-f^{\prime \prime}(x)
$$

and $M_{1} \geq M_{0}$ is an evaluation of $M_{0}$.

\section{Upper Bounding Piecewise Concave Function}

Theorem 1. Let

$$
f \in C^{2}([a, b], R \quad-0.9 e x 0.15 e x 1.4 e x \quad 0.9 e x)
$$

and suppose that there is a positive constant $M$ such that

$$
M \geq \sup _{x \in[a, b]}-f^{\prime \prime}(x) .
$$

Let

$$
P(x)=\frac{b-x}{b-a} f(a)+\frac{x-a}{b-a} f(b)
$$

and

$$
\Phi(x)=P(x)+\frac{M}{2}(x-a)(b-x) .
$$

Then

$$
\Phi(x) \geq f(x) \text { for all } x \in[a, b] .
$$

Proof. Let

$$
\Psi(x)=\Phi(x)-f(x),
$$

we have

$$
\Psi "(x)=-M-f^{\prime \prime}(x) \leq 0 .
$$

Then $\Psi$ is a concave function, the minimum of $\Psi$ over $[a, b]$ occurs at $a$ or $b$. Then

$$
\begin{aligned}
\Psi(x) & \geq \Psi(a) \\
& =\Psi(b) \\
& =0
\end{aligned}
$$

for all $x \in[a, b]$.

This proves the theorem.

Remark 1. Let

$$
u=\frac{a+b}{2}+\frac{1}{M} \frac{f(b)-f(a)}{b-a} .
$$

Then the maximum $\Phi^{*}$ of $\Phi(x)$ is:

$$
\Phi^{*}=\left\{\begin{array}{l}
\frac{1}{2}\left[f(b)+f(a)+\frac{1}{M}\left(\frac{f(b)-f(a)}{b-a}\right)^{2}\right. \\
\left.\quad+\frac{1}{4} M(a-b)^{2}\right] \\
\text { if } u \in[a, b] \\
f(a) \\
f(b)
\end{array}\right.
$$

If the function $f$ is not differentiable, we generalize the above result by the following one:

Theorem 2. Let $f$ be a continuous on $[a, b]$ and suppose that there is a positive constant $M$ such that for every $h>0$

$$
f(u+h)-2 f(u)+f(u-h) \geq M h^{2} \quad \forall u \in[a+h, b-h] .
$$

Then

$$
\begin{aligned}
f(x) \leq & \frac{b-x}{b-a} f(a)+\frac{x-a}{b-a} f(b) \\
& +\frac{M}{2}(x-a)(b-x) \quad \forall x \in[a, b] .
\end{aligned}
$$

Proof. without loss of generality, we assume that $f(a)$ $=f(b)=0$ and $M=0$.

Let us consider the function $f(x)-\Phi(x)$ instead of the function $f(x)$. It suffice to prove that

$$
f^{*}=\max _{x \in[a, b]} f(x) \leq 0 .
$$

Suppose by contradiction that $f^{*}>0$, and let

$$
u=\sup \left\{x \in[a, b] \mid f(x)=f^{*}\right\} .
$$

The function $f$ is continuous, $f(u)=f^{*}>0$, thus $a<u<b$, consequently $u \in[a+h, b-h]$ for $h>0$ small and we have $f(u-h) \leq f(u)$ and $f(u+h)<f(u)$.

Since $M=0$, this contradicts the hypothesis (3). Hence $f^{*} \leq 0$.

Remark 2. Since the above algorithm is based entirely on the result of Theorem 1, it is clear that the same algorithm will be adopted for functions satisfying condition (2).

If $f$ is twice continuously differentiable, the conditions (1) and (2) are equivalents.

\section{Modified Piyavskii's Algorithm}

We call subproblem the set of information

$$
P_{i}=\left[\Phi_{i}^{*}, x_{i}^{*}, a_{i}, b_{i}\right],
$$

describing the bounding concave function 


$$
\begin{gathered}
\Phi_{i}(x)=\frac{\left(b_{i}-x\right) f\left(a_{i}\right)+\left(x-a_{i}\right) f\left(b_{i}\right)}{b_{i}-a_{i}} \\
+\frac{M}{2}\left(x-a_{i}\right)\left(b_{i}-x\right), \\
\Phi_{i}^{*}=\max _{x \in\left[a_{i}, b_{i}\right]} \Phi_{i}(x) \text { and } x_{i}^{*}=\arg \max _{x \in\left[a_{i}, b_{i}\right]} \Phi_{i}(x) .
\end{gathered}
$$

The algorithm that we propose memorizes all the subproblems and, in particular, stores the maximum $\Phi_{i}^{*}$ of each bounding concave function $\Phi_{i}$ over $\left[a_{i}, b_{i}\right]$ in a structure called Heap. A Heap is a data structure that allows an access to the maximum of the $k$ values that it stores in constant time and is updated in $O\left(\log _{2} k\right)$ time. In the following discussion, we denote the Heap by $H$.

Modified Piyavskii's algorithm can be described as follows:

\subsection{Step 1 (Initialization)}

- $k \leftarrow 0$

- $\left[a_{0}, b_{0}\right] \leftarrow[a, b]$

- Compute $f\left(a_{0}\right)$ and $f\left(b_{0}\right)$

- $f_{\text {opt }}^{0} \leftarrow \max \left\{f\left(a_{0}\right), f\left(b_{0}\right)\right\}$

- $x_{\text {opt }}^{0} \leftarrow \operatorname{argmax}\left\{f\left(a_{0}\right), f\left(b_{0}\right)\right\}$

- Let $\Phi_{0}$ be an upper bounding function of $f$ over $\left[a_{0}\right.$, $\left.b_{0}\right]$

- Compute $\Phi_{0}^{*}=\max _{x \in\left[a_{0}, b_{0}\right]} \Phi_{0}(x)$

- $\Phi_{\text {opt }}^{0} \leftarrow \Phi_{0}^{*}$

- if $\Phi_{o p t}^{0}-f_{o p t}^{0} \leq \varepsilon$ Stop, $f_{o p t}^{0}$ is an $\varepsilon$-optimal value

- $H \leftarrow\left\{P_{0}=\left[\Phi_{0}^{*}, x_{0}^{*}, a_{0}, b_{0}\right]\right\}$

\subsection{Current Step $(k=1,2, \cdots)$}

While $H$ is no empty do

- $k \leftarrow k+1$

- Extract from $H$ the subproblem $P_{m}=\left[\Phi_{m}^{*}, x_{m}^{*}, a_{m}, b_{m}\right]$ with the largest upper bound $\Phi_{l}^{*}$

- $\left[a_{k}, b_{k}\right] \leftarrow\left[x_{m}^{*}, b_{m}\right]$

- $\left[a_{l}, b_{l}\right] \leftarrow\left[a_{m}, x_{m}^{*}\right]$

\subsubsection{Update of the Best Current Solution for $j=l, k$ do}

\subsubsection{Lower Bound}

$$
\text { If } f\left(x_{j}^{*}\right)>f_{o p t}^{k-1} \text { then }
$$

$$
\begin{aligned}
& f_{o p t}^{k} \leftarrow f\left(x_{j}^{*}\right) \\
& x_{o p t}^{k} \leftarrow x_{j}^{*}
\end{aligned}
$$

Else

$$
\begin{aligned}
& f_{o p t}^{k} \leftarrow f_{o p t}^{k-1} \\
& x_{o p t}^{k} \leftarrow x_{o p t}^{k-1}
\end{aligned}
$$

\section{End if}

\subsubsection{Upper Bound}

- Build an upper bounding function $\Phi_{j}$ on $\left[a_{j}, b_{j}\right]$

- Compute $\Phi_{j}^{*}=\max _{x \in\left[a_{j}, b_{j}\right]} \Phi_{j}(x)$

- Add $P_{j}=\left[\Phi_{j}^{*}, x_{j}^{*}, a_{j}, b_{j}\right]$ to $H$

\section{End for}

- Delete from $H$ all subproblems

$$
P=\left[\Phi_{p}^{*}, x_{p}^{*}, a_{p}, b_{p}\right]
$$

with $\Phi_{p}^{*} \leq f_{o p t}^{k}$

\subsubsection{Optimality Test}

- Let $\Phi_{o p t}^{k}$ be the maximum of all $\Phi_{j}^{*}$

- If $\Phi_{o p t}^{k}-f_{o p t}^{k} \leq \varepsilon$, then Stop, $f_{o p t}^{k}$ is an $\varepsilon$-optimal value

\section{End While}

Let $\left[a_{n}, b_{n}\right]$ denote the smallest subinterval of $[a, b]$ containing $x_{n}$, whose end points are evaluation points. Then the partial upper bounding function $\Phi_{n-1}(x)$ spanning $\left[a_{n}, b_{n}\right]$ is deleted and replaced by two partial upper bounding functions, the first one spanning $\left[a_{n}, x_{n}\right]$ denoted by $\Phi_{n l}(x)$ and the second one spanning $\left[x_{n}, b_{n}\right]$ denoted by $\Phi_{n r}(x)$ (Figure 1).

Proposition 1. For $n \geq 2$ the upper bounding function $\Phi_{n}(x)$ is easily deduced from $\Phi_{1}(x), \cdots, \Phi_{n-1}(x)$ as follows:

$$
\Phi_{n l}^{*}=\frac{\left(\Phi_{n-1}^{*}+f\left(x_{n}\right)\right)^{2}-4 f\left(a_{n}\right)\left(x_{n}\right)}{4\left(\Phi_{n-1}^{*}-f\left(a_{n}\right)\right)}
$$

and

$$
\Phi_{n r}^{*}=\frac{\left(\Phi_{n-1}^{*}+f\left(x_{n}\right)\right)^{2}-4 f\left(b_{n}\right) f\left(x_{n}\right)}{4\left(\Phi_{n-1}^{*}-f\left(b_{n}\right)\right)} .
$$

Proof. In the case where

$$
x^{\prime}=\frac{a_{n}+x_{n}}{2}+\frac{1}{M} \frac{f\left(x_{n}\right)-f\left(a_{n}\right)}{x_{n}-a_{n}}
$$




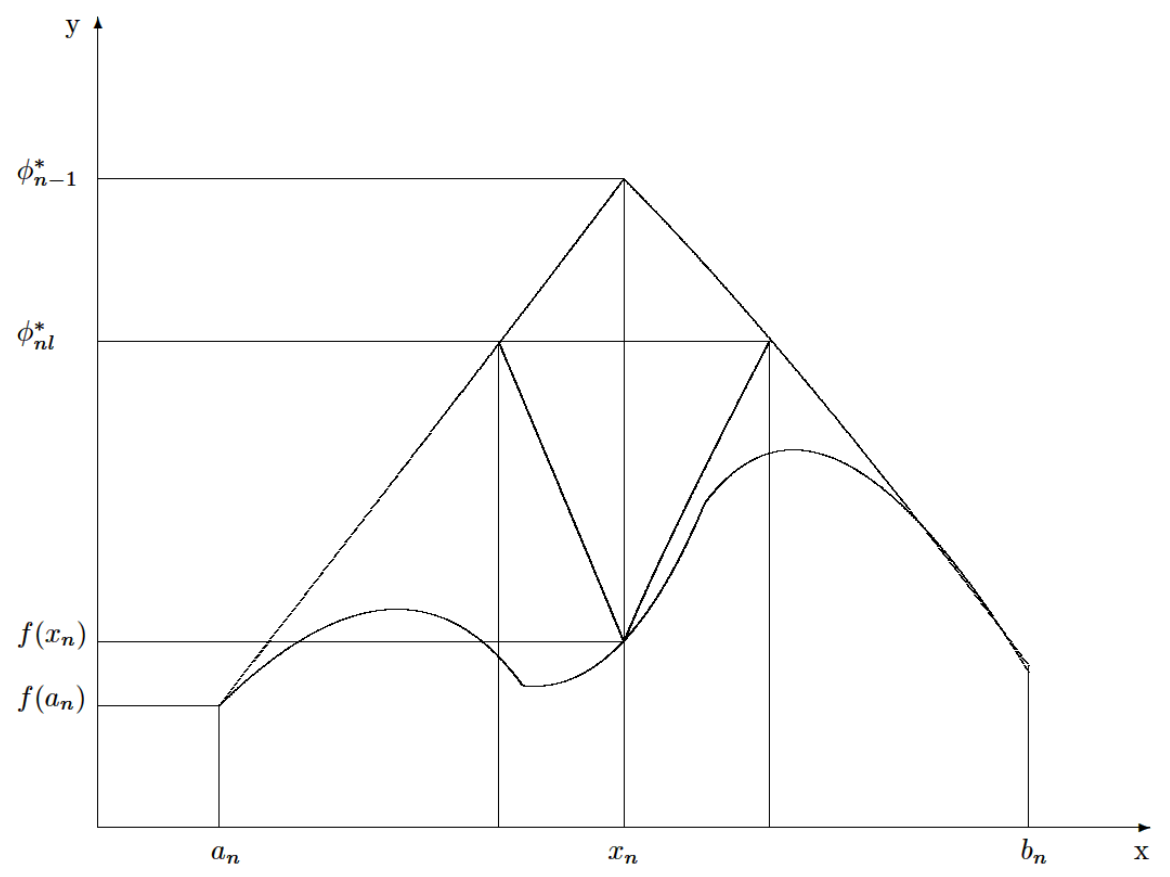

Figure 1. Upper bounding piece-wise concave function.

is in $\left[a_{n}, x_{n}\right]$, then from remark 1 , the maximum of $\Phi_{n l}(x)$ is given as follows

$$
\begin{aligned}
\Phi_{n l}^{*}=\frac{1}{2}\left[f\left(x_{n}\right)+f\left(a_{n}\right)+\frac{1}{M}\left(\frac{f\left(x_{n}\right)-f\left(a_{n}\right)}{x_{n}-a_{n}}\right)^{2}\right. \\
\left.+\frac{1}{4} M\left(x_{n}-a_{n}\right)^{2}\right]
\end{aligned}
$$

The maximum of $\Phi_{n-1}(x)$ spanning $\left[a_{n}, b_{n}\right]$ is reached at the point

$$
x_{n}=\frac{a_{n}+b_{n}}{2}+\frac{1}{M} \frac{f\left(b_{n}\right)-f\left(a_{n}\right)}{b_{n}-a_{n}} .
$$

We deduce that

$$
\begin{aligned}
\left(x_{n}-a_{n}\right)^{2}= & \frac{1}{4}\left(b_{n}-a_{n}\right)^{2}+\frac{1}{M}\left(f\left(b_{n}\right)-f\left(a_{n}\right)\right) \\
& +\frac{1}{M^{2}}\left(\frac{f\left(b_{n}\right)-f\left(a_{n}\right)}{b_{n}-a_{n}}\right)^{2},
\end{aligned}
$$

then

$$
\frac{1}{4} M\left(x_{n}-a_{n}\right)^{2}=\frac{1}{2}\left(\Phi_{n-1}^{*}-f\left(a_{n}\right)\right) .
$$

By substitution in expression (3), we have the result. We show in the same way that the maximum $\Phi_{n r}^{*}$ of $\Phi_{n r}(x)$ defined in $\left[x_{n}, b_{n}\right]$ is given by:

$$
\Phi_{n r}^{*}=\frac{\left(\Phi_{n-1}^{*}+f\left(x_{n}\right)\right)^{2}-4 f\left(b_{n}\right) f\left(x_{n}\right)}{4\left(\Phi_{n-1}^{*}-f\left(b_{n}\right)\right)} .
$$

Remark 3. Modified Piyavskii's algorithm obtains an upper bound on $f$ within $\varepsilon$ of the maximum value of $f(x)$ when the gap $\Phi_{n}^{*}-f^{*}$ is not larger than $\varepsilon$, where

$$
f^{*}=\max _{x \in[a, b]} f(x) .
$$

Butf* and

$$
x^{*}=\arg \max _{x \in[a, b]} f(x)
$$

are unknown. So modified Piyavskii's algorithm stops only after a solution of value within $\varepsilon$ of the upper bound has been found, i.e., when the error $\Phi_{n}^{*}-f_{n}^{*}$ is not larger than $\varepsilon$. The number of iterations needed to satisfy each of these conditions is studied below.

\section{Convergence of the Algorithm}

We now study the error and the gap of $\Phi_{n}$ in modified Piyavskii's algorithm as a function of the number $n$ of iterations. The following proposition shows how they decrease when the number of iterations increases and provides also a relationship between them.

Proposition 2. Let $\delta_{n}=\Phi_{n}^{*}-f_{n}^{*}$ and $\delta_{n}^{\prime}=\Phi_{n}^{*}-f^{*}$ denote respectively the error and the gap after $n$ iterations of modified Piyavskii's algorithm. Then, for $n \geq 2$ 1) $\delta_{2 n-1} \leq \delta_{n} / 4$, 2) $\delta_{2 n-1}^{\prime} \leq \delta_{n}^{\prime} / 4$, 3) $\delta_{2 n-1} \leq \delta_{n}^{\prime} / 2$.

Proof. First, notice that $\left(\Phi_{n}^{*}\right),\left(\delta_{n}\right)$ and $\left(\delta_{n}^{\prime}\right)$ are non increasing sequences and $\left(f_{n}^{*}\right)$ is nondecreasing. After $n$ iterations of the modified Piyavskii's algorithm, the upper bounding piece-wise concave function $\Phi_{n}(x)$ contains $(n-1)$ partial upper bounding concave functions, which we call first generation function. We call the partial upper bounding concave functions obtained by 
splitting these second generation function. $\Phi_{m}$ must belong to the second generation for the first time after no more than another $n-1$ iterations of the modified Piyavskii's algorithm, at iteration $m(m \leq 2 n-1)$.

Let $k(n+1 \leq k \leq m)$ be the iteration after which the maximum $\Phi_{m}^{*}$ of $\Phi_{m}(x)$ has been obtained, by splitting the maximum $\Phi_{k-1}^{*}$ of $\Phi_{k-1}(x)$. Then, in the case where

$$
x_{k}^{\prime}=\frac{a_{k}+x_{k}}{2}+\frac{f\left(x_{k}\right)-f\left(a_{k}\right)}{M\left(x_{k}-a_{k}\right)}
$$

is in $\left[a_{k}, x_{k}\right]$, and from proposition 1, we get:

$$
\begin{aligned}
\Phi_{m}^{*} & =\frac{\left(\Phi_{k-1}^{*}+f\left(x_{k}\right)\right)^{2}-4 f\left(a_{k}\right) f\left(x_{k}\right)}{4\left(\Phi_{k-1}^{*}-f\left(a_{k}\right)\right)} \\
& =\frac{1}{4}\left\{2 f\left(x_{k}\right)+f\left(a_{k}\right)+\Phi_{k-1}^{*}+\frac{\left(f\left(x_{k}\right)-f\left(a_{k}\right)\right)^{2}}{\Phi_{k-1}^{*}-f\left(a_{k}\right)}\right\},
\end{aligned}
$$

where

$$
x_{k}=\arg \max _{x \in[a, b]} \Phi_{k-1}(x)
$$

and $a_{k}$ is one of the endpoints of the interval over which the function $\Phi_{k-1}$ is defined.

1) We have

$$
\delta_{2 n-1}=\Phi_{2 n-1}^{*}-f_{2 n-1}^{*} \leq \Phi_{m}^{*}-f_{m}^{*},
$$

where

$$
f_{m}^{*}=\max _{i=0, \cdots, m} f\left(x_{i}\right)=\max \left(f_{k-1}^{*}, f\left(x_{k}\right)\right) .
$$

If $f_{m}^{*}=f\left(x_{k}\right)$, therefore, we have

$$
\begin{aligned}
& \leq \frac{\left(\Phi_{k-1}^{*}+f\left(x_{k}\right)\right)^{2}-4 f\left(a_{k}\right) f\left(x_{k}\right)}{4\left(\Phi_{k-1}^{*}-f\left(a_{k}\right)\right)}-f\left(x_{k}\right) \\
& \leq \frac{\left(\Phi_{k-1}^{*}-f\left(x_{k}\right)\right)^{2}}{4\left(\Phi_{k-1}^{*}-f\left(a_{k}\right)\right)} \\
& \leq \frac{\Phi_{k-1}^{*}-f\left(x_{k}\right)}{4} \\
& \leq \frac{\Phi_{n}^{*}-f_{n}^{*}}{4} \\
& =\frac{\delta_{n}}{4} .
\end{aligned}
$$

If $f_{m}^{*}=f_{k-1}^{*}$, we will consider two cases:

\section{- case 1}

If $x_{k}^{\prime} \leq a_{k}$, then $\Phi_{m}^{*}=f\left(a_{k}\right)=f_{m}^{*}$ and if $x_{k}^{\prime} \geq x_{k}$, then $\Phi_{m}^{*}=f\left(x_{k}\right)=f_{m}^{*}$ and the algorithm stops.

- case 2 $x_{k}^{\prime} \in\left[a_{k}, x_{k}\right]$, then we have

$$
x_{k}^{\prime}=\frac{a_{k}+x_{k}}{2}+\frac{1}{M} \frac{f\left(x_{k}\right)-f\left(a_{k}\right)}{x_{k}-a_{k}} \geq a_{k},
$$

hence

$$
\begin{aligned}
f\left(a_{k}\right)-f\left(x_{k}\right) & \leq \frac{M}{2}\left(x_{k}-a_{k}\right)^{2} \\
& =\Phi_{k-1}^{*}-f\left(a_{k}\right)
\end{aligned}
$$

(proposition 1).

Moreover

$$
f\left(x_{k}\right)-f\left(a_{k}\right) \leq \Phi_{k-1}^{*}-f\left(a_{k}\right) .
$$

We deduce from these two inequalities that

$$
\left|f\left(x_{k}\right)-f\left(a_{k}\right)\right| \leq \Phi_{k-1}^{*}-f\left(a_{k}\right) .
$$

Therefore

$$
\delta_{2 n-1}
$$$$
\leq \frac{1}{4}\left\{2 f\left(x_{k}\right)+f\left(a_{k}\right)+\Phi_{k-1}^{*}+\frac{\left(f\left(x_{k}\right)-f\left(a_{k}\right)\right)^{2}}{\Phi_{k-1}^{*}-f\left(a_{k}\right)}\right\}-f_{k-1}^{*}
$$$$
\leq \frac{1}{4}\left\{2 f\left(x_{k}\right)+\Phi_{k-1}^{*}+f\left(a_{k}\right)+\left|f\left(x_{k}\right)-f\left(a_{k}\right)\right|\right\}-f_{k-1}^{*}
$$$$
\leq \frac{1}{4}\left(3 f_{k-1}^{*}+\Phi_{k-1}^{*}\right)-f_{k-1}^{*}
$$

$\leq \frac{\Phi_{k-1}^{*}-f_{k-1}^{*}}{4}$

$\leq \frac{\Phi_{n}^{*}-f_{n}^{*}}{4}=\delta_{n} / 4$.

2) We have

$$
\delta_{2 n-1}^{\prime}=\Phi_{2 n-1}^{*}-f^{*} \leq \Phi_{m}^{*}-f^{*} .
$$

We follow the same steps to prove the second point of 1) and show that $\delta_{2 n-1}^{\prime} \leq \delta_{n}^{\prime} / 4$.

3)

- case 1

If $x_{k}^{\prime} \leq a_{k}$ or $x_{k}^{\prime} \geq x_{k}$, then $\Phi_{m}^{*}=f_{m}^{*}=f^{*}$ and the algorithm stops.

- case $2 x_{k}^{\prime} \in\left[a_{k}, x_{k}\right]$. First, we have

$$
\begin{aligned}
\Phi_{m}^{*} & =\frac{1}{4}\left\{2 f\left(x_{k}\right)+f\left(a_{k}\right)+\Phi_{k-1}^{*}+\frac{\left(f\left(x_{k}\right)-f\left(a_{k}\right)\right)^{2}}{\Phi_{k-1}^{*}-f\left(a_{k}\right)}\right\} \\
& \leq \frac{f\left(x_{k}\right)+\Phi_{k-1}^{*}}{2},
\end{aligned}
$$

and 


$$
\begin{aligned}
f^{*} & \leq \Phi_{m}^{*} \\
& \leq \frac{f\left(x_{k}\right)+\Phi_{k-1}^{*}}{2} \\
& \leq \frac{f^{*}+\delta_{n}^{\prime}+f\left(x_{k}\right)}{2},
\end{aligned}
$$

therefore $f\left(x_{k}\right) \geq f^{*}-\delta_{n}^{\prime}$. Hence

$$
\delta_{2 n-1} \leq \frac{\Phi_{n}^{*}-f\left(x_{k}\right)}{4} \leq \delta_{n}^{\prime} / 2 .
$$

This proves the proposition.

Proposition 3. Modified Piyavskii's algorithm (with $\varepsilon=0)$ is convergent, i.e. either it terminates in a finite number of iterations or

$$
\lim _{n \rightarrow \infty} \Phi_{n}^{*}=\lim _{n \rightarrow \infty} f_{n}^{*}=f^{*}=\max _{x \in[a, b]} f(x) .
$$

Proof. This is an immediate consequence of the definition of $\delta_{n}$ and i) of proposition 2 .

\section{Reference Algorithm}

Since the number of necessary function evaluations for solving problem $(P)$ measures the efficiency of a method, Danilin [25] and Hansen, Jaumard and Lu [26] suggested studying the minimum number of evaluation points required to obtain a guaranteed solution of problem $(P)$ where the functions involved are Lipschitz-Continuous on $[a, b]$.

In the same way, we propose to study the minimum number of evaluation points required to obtain a guaranteed solution of problem $(P)$ where the functions involved satisfy the condition (1).

For a given tolerance $\varepsilon$ and constant $M$ this can be done by constructing a reference bounding piece-wise concave function $\Phi_{r e f}$ such that

$$
\max _{x \in[a, b]} \Phi_{r e f}(x)=f^{*}+\varepsilon,
$$

with a smallest possible number $n_{r e f}$ of evaluations points $y_{1}, y_{2}, \cdots, y_{n_{r e f}}$ in $[a, b]$.

Such a reference bounding piece-wise concave function is constructed with $f^{*}=\max _{x \in[a, b]} f(x)$ which is assumed to be known. It is of course, designed not to solve problem $(P)$ from the outset, but rather to give a reference number of necessary evaluation points in order to study the efficiency of other algorithms. It is easy to see that a reference bounding function $\Phi_{r e f}(x)$ can be obtained in the following way:

\section{Description of the Reference Algorithm}

\section{1). Initialization}

- $k \leftarrow 1$

- $y_{1} \leftarrow$ root of the equation

$$
f^{*}+\varepsilon-\frac{M}{2}(a-x)^{2}-f(x)=0 .
$$

\section{2). Reference bounding function}

While $y_{k}<b$ do

- compute $f\left(y_{k}\right)$

- $x_{p_{k}} \leftarrow y_{k}+\sqrt{\frac{2}{M}\left(f^{*}+\varepsilon-f\left(\left(y_{k}\right)\right)\right.}$

- $y_{k+1} \leftarrow$ root of the equation

$$
f^{*}+\varepsilon-f(x)-\frac{M}{2}\left(x_{p_{k}}-x\right)^{2}=0
$$

- $k \leftarrow k+1$

\section{End While.}

\section{3). Minimum number of evaluations points}

$$
\text { - } n_{\text {ref }} \leftarrow k
$$

A reference bounding function $\Phi_{r e f}$ is then defined as follows (see Figure 2)

$$
\Phi_{r e f}(x)= \begin{cases}f^{*}+\varepsilon-\frac{M}{2}(x-a)^{2} & \text { if } x \in\left[a, y_{1}\right] \\ f^{*}+\varepsilon-\frac{M}{2}\left(x-x_{p_{k}}\right)^{2} & \text { if } x \in\left[y_{k}, y_{k+1}\right], \\ f^{*}+\varepsilon-\frac{M}{2}\left(x-x_{p_{n_{r e f}}}\right)^{2} & \text { if } x \in\left[y_{n_{r e f}}, b\right]\end{cases}
$$

\section{Estimation of the Efficiency of the Modified Piyavskii's Algorithm}

Proposition 4. For a given a tolerance $\varepsilon$, let $\Phi_{\text {ref }}$ be a reference bounding function with $n_{\text {ref }}$ evaluations points $y_{1}, \cdots, y_{n_{r e f}}$ and $\Phi_{\text {ref }}^{*}=f^{*}+\varepsilon$. Let $\Phi_{C}$ be a bounding function obtained by the modified Piyavskii's algorithm with evaluation points $x_{1}, \cdots, x_{n_{C}}$, where $n_{C}$ is the smallest number of iterations such that

$$
\Phi_{C}^{*}=\max _{x \in[a, b]} \Phi_{C}(x) \leq f^{*}+\varepsilon .
$$

Then we have

$$
n_{C} \leq 2 n_{r e f}+1,
$$

and the bound is sharp for all values of $n_{r e f}$

Proof. The proof is by induction on $n_{r e f}$ We initialize the induction at $n_{r e f}=1$.

- Case $1 y_{1}=b$. (see Figure 3)

We have to show that $\Phi_{C}(x) \leq \Phi_{R}(x), \quad \forall x \in[a, b]$. We have

$$
\begin{aligned}
\Phi_{C}(x)= & \frac{(b-x) f(a)+(x-a) f(b)}{b-a} \\
& +\frac{M}{2}(x-a)(b-x)
\end{aligned}
$$




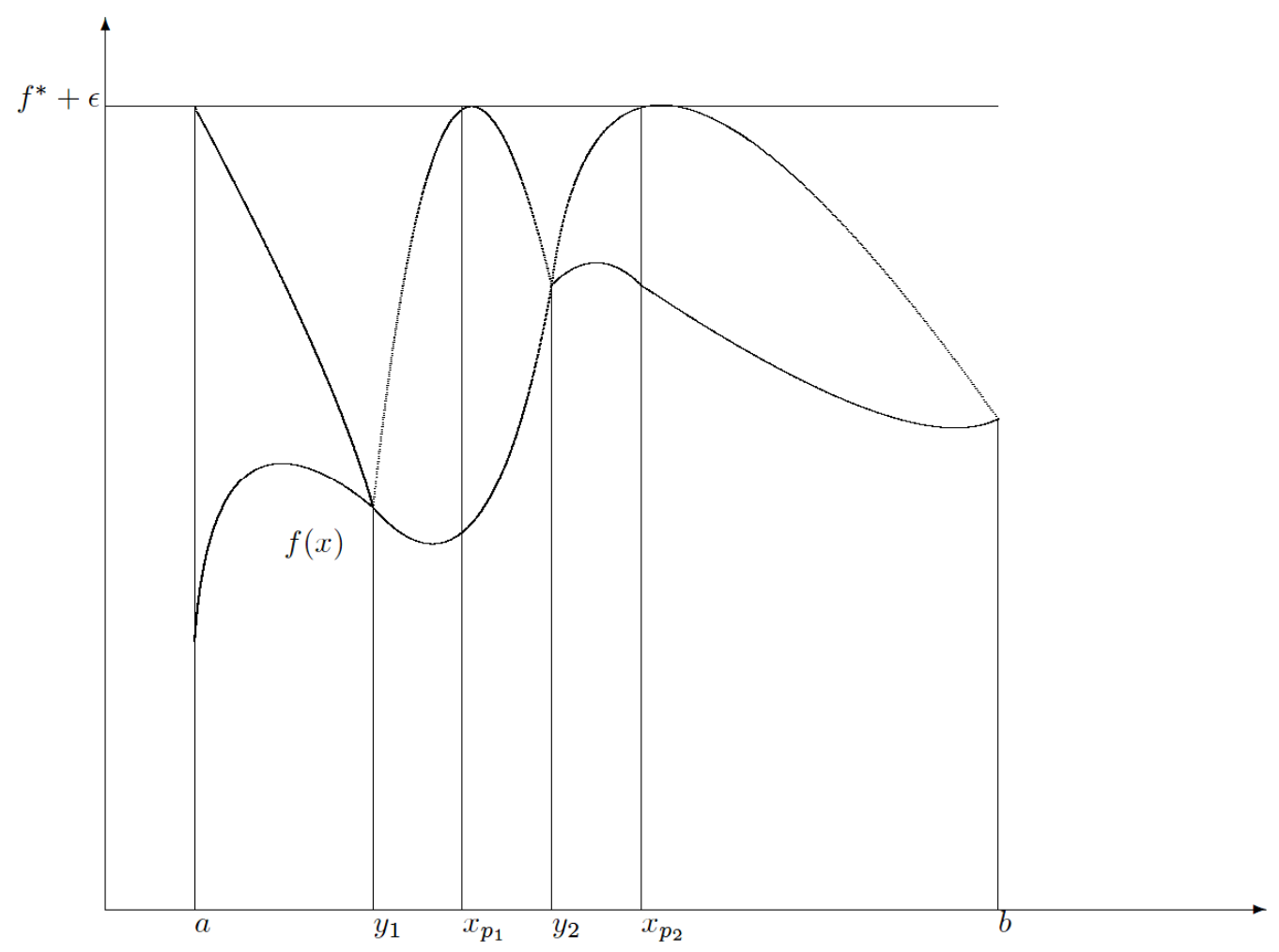

Figure 2. Reference bounding function.

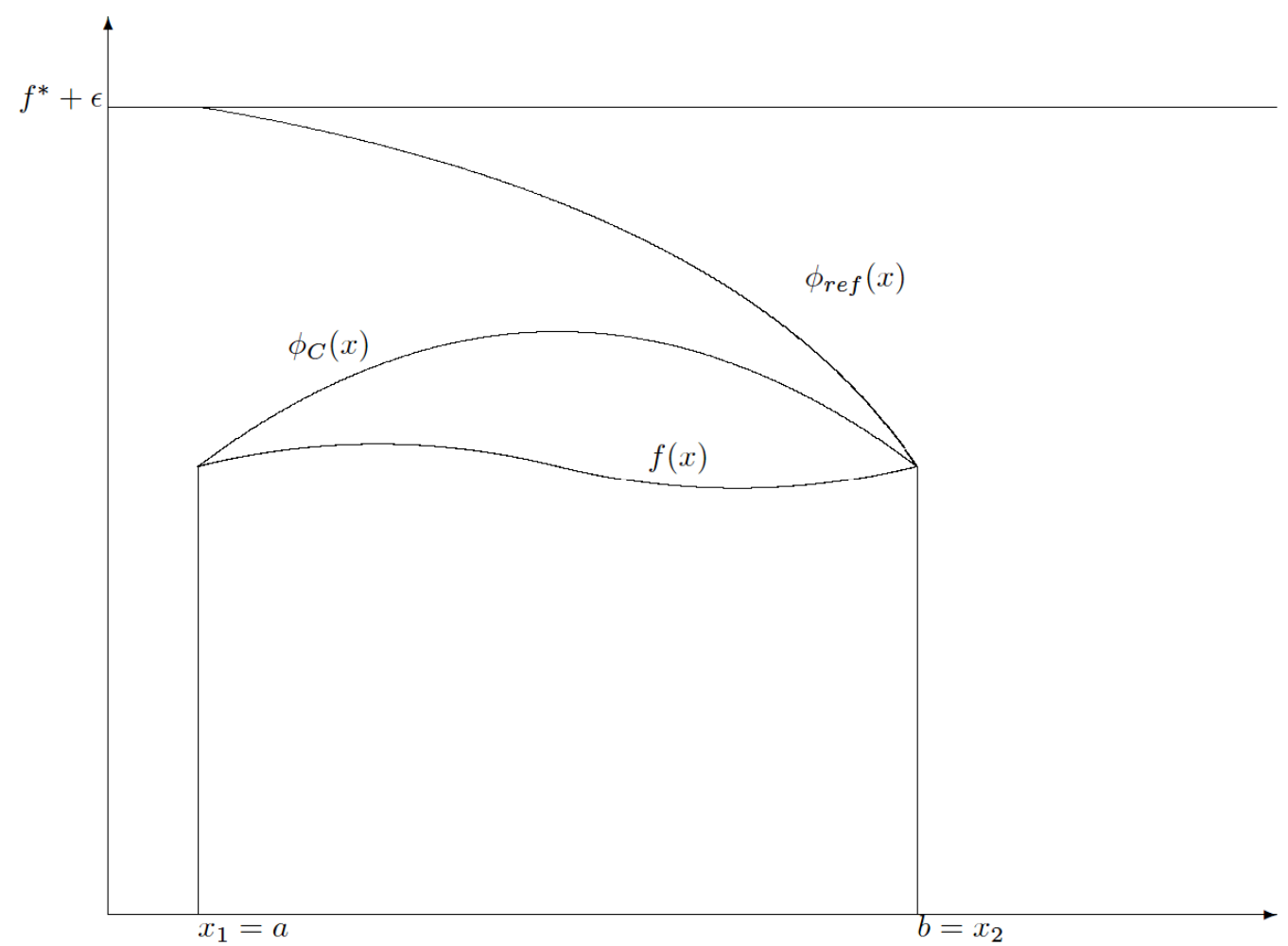

Figure 3. Case $y_{1}=b$.

and

$$
\Phi_{r e f}(x)=f^{*}+\varepsilon-\frac{M}{2}(x-a)^{2} .
$$

But

$$
f(b)=\Phi_{r e f}(b)=f^{*}+\varepsilon-\frac{M}{2}(b-a)^{2},
$$


hence

$$
\begin{aligned}
\Phi_{C}(x)= & \frac{(b-x) f(a)+(x-a)\left(f^{*}+\varepsilon\right)}{b-a} \\
& -\frac{M}{2}(x-a)(b-a)+\frac{M}{2}(x-a)(b-x) \\
= & \frac{(b-x) f(a)+(x-a)\left(f^{*}+\varepsilon\right)}{b-a}-\frac{M}{2}(x-a)^{2} .
\end{aligned}
$$

Therefore

$$
\Phi_{C}(x)-\Phi_{r e f}(x)=\frac{b-x}{b-a}\left(f(a)-\left(f^{*}+\varepsilon\right)\right) \leq 0,
$$

hence the result holds.

- case $2 y_{1}<b$. (see Figure 4)

If $n_{c}=2$, (4) holds. In this case, we have

$$
\Phi_{r e f}(x)= \begin{cases}f^{*}+\varepsilon-\frac{M}{2}(x-a)^{2} & \text { si } x \in\left[a, y_{1}\right] \\ f^{*}+\varepsilon-\frac{M}{2}\left(x-x_{p_{1}}\right)^{2} & \text { si } x \in\left[y_{1}, b\right],\end{cases}
$$

where

$$
x_{p_{1}}=y_{1}+\sqrt{\frac{2}{M}\left(f^{*}+\varepsilon-f\left(y_{1}\right)\right)} .
$$

Assuming that $\Phi_{1}^{*}>f^{*}+\varepsilon$ and $x_{3} \in\left[a, y_{1}\right]$ ( the proof is similar for $\left.x_{3} \in\left[y_{1}, b\right]\right)$. We denote by $\Phi_{l}(x)$ and $\Phi_{r}(x)$ the partial bounding functions defined respectively on $\left[a, x_{3}\right]$ and $\left[x_{3}, b\right]$ and by $\Phi_{r}^{*}$ a maximum value of $\Phi_{r}(x)$. We show that

$$
\Phi_{l}(x) \leq \Phi_{r e f}(x) \quad \forall x \in\left[a, x_{3}\right]
$$

and that $\Phi_{r}^{*} \leq f^{*}+\varepsilon$. Indeed, we have

$$
\begin{aligned}
\Phi_{l}(x)= & \frac{\left(x_{3}-x\right) f(a)+(x-a) f\left(x_{3}\right)}{x_{3}-a} \\
& +\frac{M}{2}(x-a)\left(x_{3}-x\right)
\end{aligned}
$$

and

$$
f\left(x_{3}\right) \leq \Phi_{r e f}\left(x_{3}\right)=f^{*}+\varepsilon-\frac{M}{2}\left(a-x_{3}\right)^{2},
$$

thus

$$
\begin{aligned}
& \Phi_{l}(x)-\Phi_{r e f}(x) \\
\leq & \frac{x_{3}-x}{x_{3}-a}\left(f(a)-\left(f^{*}+\varepsilon\right)\right) \\
\leq & 0 \quad \forall x \in\left[a, x_{3}\right] .
\end{aligned}
$$

If

$$
x^{\prime \prime}=\frac{x_{3}+b}{2}+\frac{f(b)-f\left(x_{3}\right)}{M\left(b-x_{3}\right)}
$$

is in $\left[x_{3}, b\right]$, then

$$
\Phi_{r}^{*}=\frac{1}{2}\left\{f\left(x_{3}\right)+\frac{1}{2}\left(f(b)+\Phi_{1}^{*}\right)+\frac{\left(f(b)-f\left(x_{3}\right)\right)^{2}}{2\left(\Phi_{1}^{*}-f(b)\right)}\right\} .
$$

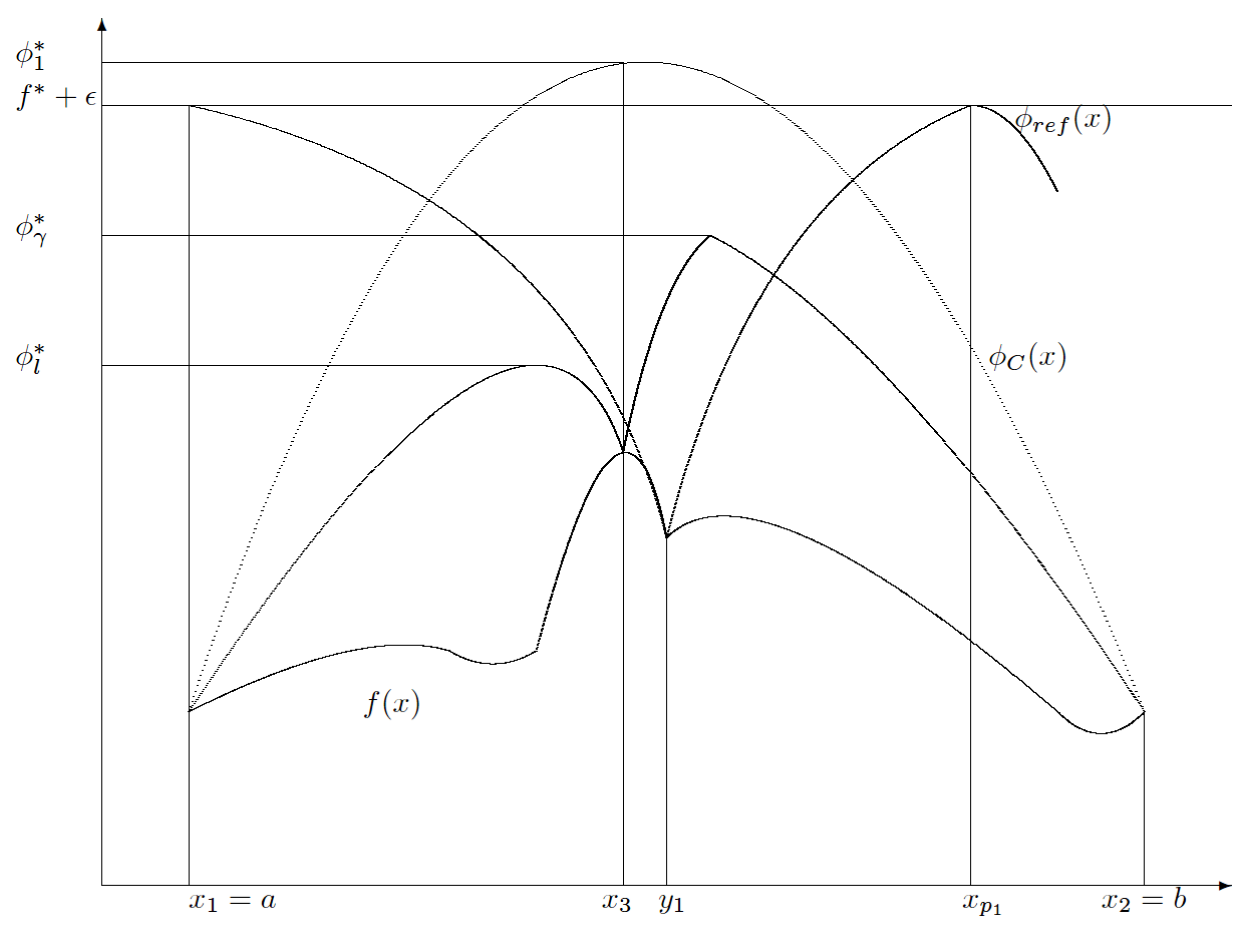

Figure 4. Case $y_{1}<b$. 
As

$$
\left|f(b)-f\left(x_{3}\right)\right| \leq \Phi_{1}^{*}-f(b)
$$

(see the proof of proposition 2), we have

$$
\begin{aligned}
\Phi_{r}^{*} & \leq \frac{1}{2}\left\{f\left(x_{3}\right)+\Phi_{1}^{*}\right\} \\
& \leq \frac{1}{2}\left\{f^{*}+\varepsilon-\frac{M}{2}\left(a-x_{3}\right)^{2}+\Phi_{1}^{*}\right\} \\
& \leq \frac{1}{2}\left\{f^{*}+\varepsilon-\Phi_{1}^{*}+f(a)+\Phi_{1}^{*}\right\} \leq f^{*}+\varepsilon .
\end{aligned}
$$

If $x^{\prime \prime} \geq b$, then $\Phi_{r}^{*}=f(b) \leq f^{*}+\varepsilon$.

If $x^{\prime \prime} \leq x_{3}$ then $\Phi_{r}^{*}=f\left(x_{3}\right) \leq f^{*}+\varepsilon$ and (4) holds.

Assume now that (4) holds for $n_{\text {ref }} \leq k(k \geq 1)$, and consider $n_{r e f}=k+1$. The relation (4) holds for $n_{c}=2$, therefore we may assume that

$$
\Phi_{1}^{*}>f^{*}+\varepsilon, x_{1}=a \text { and } x_{2}=b .
$$

If we let

$$
x_{3}=\arg \max _{x \in[a, b]} \Phi_{1}(x),
$$

the modified Piyavskii's algorithm has to be implemented on two subintervals $\left[x_{1}, x_{3}\right]$ and $\left[x_{3}, x_{2}\right]$ There are two cases which need to be discussed separately:

- case 1 (see Figure 5)

There is a subinterval containing all the $n_{\text {ref }}$ evaluation points of the best bounding function $\Phi_{\text {ref }}(x)$. Without loss of generality, we may assume that $\left[x_{1}, x_{3}\right]$ contains all $n_{\text {ref }}$ evaluation points of $\Phi_{r e f}$. Let $x^{\prime}$ and $x^{\prime \prime}$ be the solution of $\Phi_{1}(x)=f^{*}+\varepsilon$. By symmetry, we have

$$
x_{3}=\frac{x^{\prime}+x^{\prime \prime}}{2} .
$$

Let $y_{n_{r e}}^{\prime}$ be the abscissa of the maximum of the last partial bounding function of $\Phi_{\text {ref }}(x)$. Due to our assumption, we have

$$
y_{n_{\text {ref }}}^{\prime}=y_{n_{\text {ref }}}+\sqrt{\frac{2}{M}\left(f^{*}+\varepsilon-f\left(\left(y_{n_{\text {ref }}}\right)\right)\right.} \geq x^{\prime \prime},
$$

since otherwise there is an another evaluation point of $f$ on the interval

$$
\left[y_{n_{\text {ref }}}, b\right] .
$$

Let $y_{n_{\text {ref }}-1}^{\prime}$ be the abscissa of the maximum of the partial bounding function of $\Phi_{r e f}(x)$. preceding $y_{n_{r e f}}^{\prime}$. In this case, we have

$$
x^{\prime}>y_{n_{r e f}-1}^{\prime}=y_{n_{r e f}}-\sqrt{\frac{2}{M}\left(f^{*}+\varepsilon-f\left(y_{n_{r e f}}\right)\right)} .
$$

But $y_{n_{r e f}-1}^{\prime}<x^{\prime}$ implies that $n_{\text {ref }}=1$, which is not the case according to the assumption $\left(n_{r e f} \geq 2\right)$. Therefore, this case will never exist.

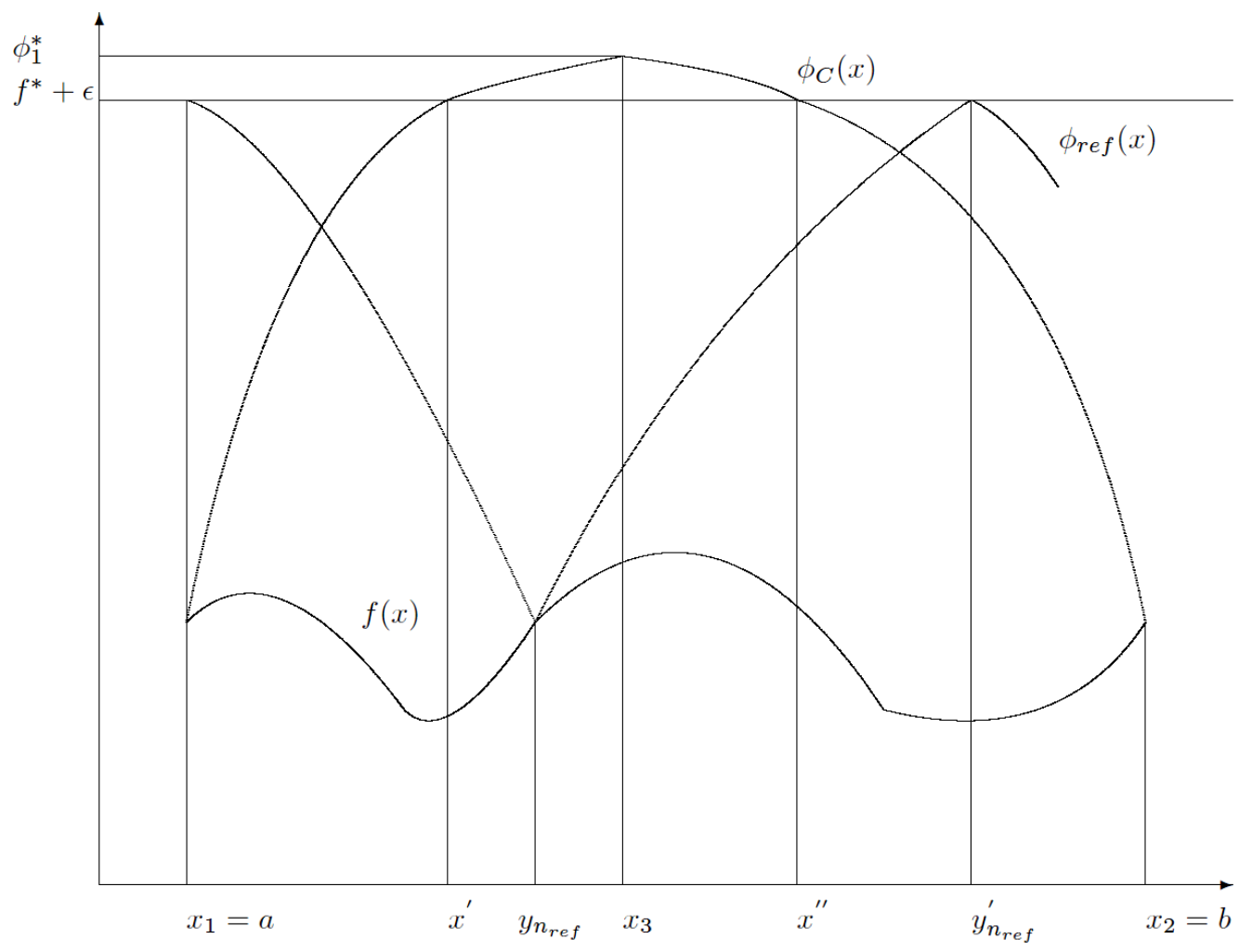

Figure 5. The subinterval contains all the $n_{r e f}$ evaluation points. 
- case 2

None of the subintervals contains all the $n_{\text {ref }}$ evaluation points of $\Phi_{r e f}(x)$. We can then apply induction, reasoning on the subintervals $\left[x_{1}, x_{3}\right]$ and $\left[x_{3}, x_{2}\right]$ obtained by modified Piyavskii's algorithm. If they contain $n_{c}^{1}$ and $n_{c}^{2}$ evaluation points respectively, $\left[x_{1}, x_{2}\right]$ contains $n_{c}^{1}+n_{c}^{2}-1$ evaluation points as $x_{3}$ belongs to both subintervals. Then (4) follows by induction on $n_{r e f}$.

Consider the function $f(x)=x$ defined on $[0,1]$, the constant $M$ is equal to 4 . For $\varepsilon=0$ we have $n_{C}=n_{\text {ref }}+1$ (see Figure 6), and the bound (4) is sharp.

As noticed in remark 3, the modified Piyavskii's algorithm does not necessarily stop as soon as a cover is found as described in proposition 4 , but only when the error does not exceed $\varepsilon$. We now study the number of evaluation points necessary for this to happen.

Proposition 5. For a given tolerance $\varepsilon$, let $n_{\text {ref }}$ be the number of evaluation points in a reference bounding function of $f$ and $n_{B}$ the number of iterations after which the modified Piyavskii's algorithm stops. We then have

$$
n_{B} \leq 4 n_{r e f}+1 \text {. }
$$

Proof. Let $n_{C^{\prime}}=2 n_{\text {ref }}+1$. Due to proposition 4, after $n_{C^{\prime}}$ iterations, we have $\Phi_{C^{\prime}}^{*}-f^{*} \leq \varepsilon$. Due to 3 ) of proposition 2 , after $2 n_{C^{\prime}}-1$ iterations, we have

$$
\Phi_{2{ }^{\prime} C^{\prime}-1}^{*}-f_{2 n_{C^{\prime}-1}}^{*} \leq \Phi_{{ }_{n^{\prime}}}^{*}-f^{*},
$$

which shows that the termination of modified Piyavskii's algorithm is satisfied. This proves (5).

\section{Bounds on the Number of Evaluation Points}

In the previous section, we compared the number of evaluation functions in the modified Piyavskii's algorithm and in the reference algorithm. We now evaluate the number $n_{B}$ of evaluation functions of the modified Piyavskii's algorithm itself. To achieve this, we derive bounds on $n_{\text {ref }}$, from which bounds on $n_{B}$ are readily obtained using the relation $n_{\text {ref }} \leq n_{B} \leq 4 n_{\text {ref }}+1$.

Proposition 6. Let $f$ be a $C^{2}$ function defined on the interval $[a, b]$. Set

$$
M_{0}=\sup _{x \in[a, b]}-f^{\prime \prime}(x)
$$

and let

$$
f^{*}=\max _{x \in[a, b]} f(x) .
$$

Then the number $n_{\text {ref }}$ of evaluation points in a reference cover $\Phi_{\text {ref }}$ using the constant $M_{1} \geq M_{2}$ is bounded by the following inequalities:

$$
n_{r e f} \leq \frac{\sqrt{\left(M_{1}+M_{0}\right)\left(f^{*}+\varepsilon\right)} \int_{a}^{b} \frac{\mathrm{d} x}{f^{*}+\varepsilon-f(x)}}{\left.\sqrt{2} \log \left(1+\sqrt{\frac{M_{0}}{M_{1}+M_{0}}}\right)\right)} .
$$

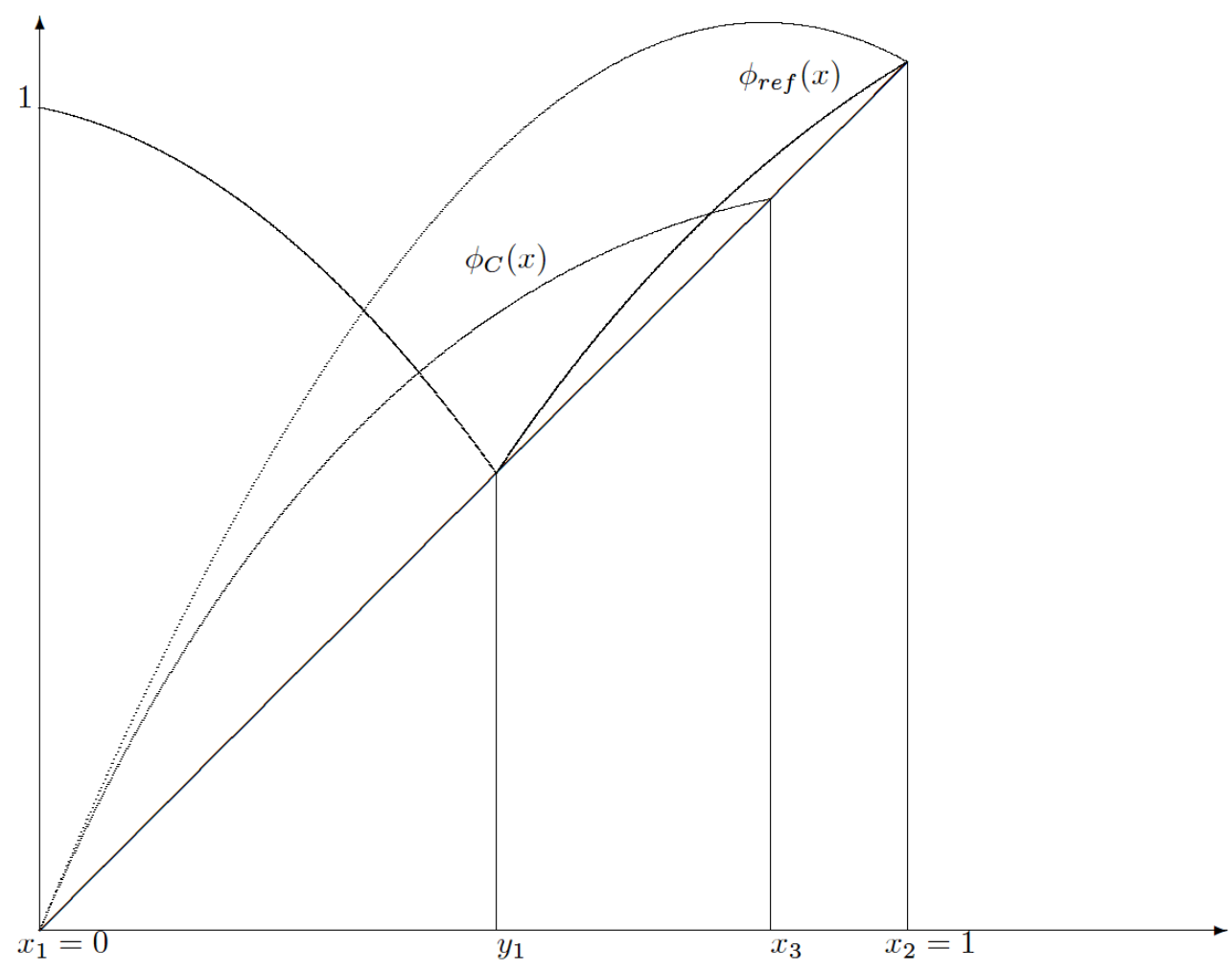

Figure 6. None of the subintervals contains all the $\boldsymbol{n}_{\text {ref }}$ evaluation points. 


$$
n_{r e f} \geq\left\{\begin{array}{l}
\frac{\sqrt{M_{0} \varepsilon}}{\pi \sqrt{2}} \int_{a}^{b} \frac{\mathrm{d} x}{f^{*}+\varepsilon-f(x)} \\
\text { 2in } .2 \text { inif }\left(\frac{M_{1}-M_{0}}{2 M_{1}}\right)^{2}\left(f^{*}+\varepsilon\right) \geq \varepsilon, \\
\frac{\sqrt{M_{0} \varepsilon}}{4 \sqrt{2}} \int_{a}^{b} \frac{\mathrm{d} x}{f^{*}+\varepsilon-f(x)} \\
\text {.2in .2inif } M_{0}=M_{1} .
\end{array}\right.
$$

Proof. We suppose that the reference cover $\Phi_{r e f}$ has $n_{\text {ref }}-1$ partial upper bounding functions defined by the evaluation points

$$
a=y_{1}, y_{2}, \cdots, y_{n_{\text {ref }}}=b .
$$

We consider an arbitrary partial upper bounding function and the corresponding subinterval $\left[y_{i}, y_{i+1}\right]$ for $i=1, \cdots$, $n_{r e f}-1$. To simplify, we move the origin to the point $\left(y_{i}\right.$, $\left.f\left(y_{i}\right)\right)$. Let $d=y_{i+1}-y_{i}, z=f\left(y_{i+1}\right)-f\left(y_{i}\right)$ and $h=f^{*}+\varepsilon$. We assume $z \geq 0$ (See Figure 7).

Let $\Phi_{r e f}^{r}$ be the partial upper bounding function defined on $\left[y_{i}, y_{i+1}\right]$ and $x_{p_{i}}$ the point where

$$
\max _{x \in\left[y_{i}, y_{i+1}\right]} \Phi_{r e f}^{r}(x)=f^{*}+\varepsilon
$$

is reached, then

$$
\begin{aligned}
x_{p_{i}} & =y_{i}+\sqrt{\frac{2}{M}\left(f^{*}+\varepsilon-f\left(y_{i}\right)\right)} \\
& =y_{i+1}-\sqrt{\frac{2}{M}\left(f^{*}+\varepsilon-f\left(y_{i+1}\right)\right)} .
\end{aligned}
$$

We deduce that

$$
h=\frac{1}{2 M_{1}}\left(\frac{z}{d}+\frac{\mathrm{d} M_{1}}{2}\right)^{2} .
$$

Let $g_{1}$ be the function defined on $\left[y_{i}, y_{i+1}\right]$ by:

$$
\begin{aligned}
& g_{1}(x) \\
= & \frac{\left(y_{i+1}-x\right) f\left(y_{i}\right)+\left(x-y_{i}\right) f\left(y_{i+1}\right)}{y_{i+1}-y_{i}} \\
& -\frac{M_{0}}{2}\left(x-y_{i}\right)\left(y_{i+1}-x\right) \\
= & \left(\frac{z}{d}-\frac{M_{0} d}{2}\right) x+\frac{M_{0}}{2} x^{2} .
\end{aligned}
$$

We have

$$
f(x) \geq g_{1}(x) \quad \forall x \in[a, b] .
$$

Thus we have

$$
\begin{aligned}
& \int_{0}^{d} \frac{\mathrm{d} x}{f^{*}+\varepsilon-f(x)} \\
\geq & \int_{0}^{d} \frac{\mathrm{d} x}{f^{*}+\varepsilon-g_{1}(x)} \\
= & \int_{0}^{d} \frac{\mathrm{d} x}{\frac{1}{2 M_{1}}\left(\frac{z}{d}+\frac{\mathrm{d} M_{1}}{2}\right)^{2}-\left(\frac{z}{d}-\frac{M_{0} d}{2}\right) x-\frac{M_{0}}{2} x^{2}} .
\end{aligned}
$$

Consider the function

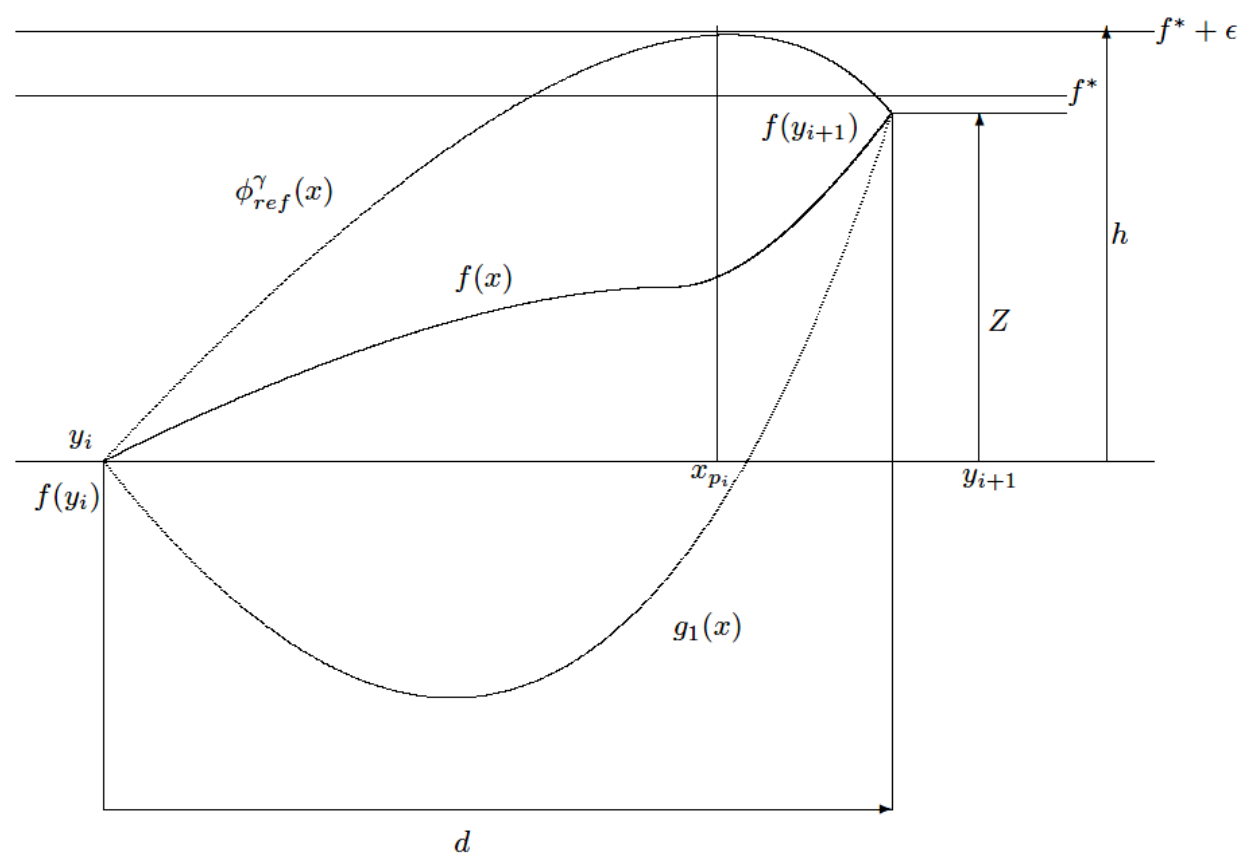

Figure 7. The reference cover $\Phi_{r e f}$ has $n_{r e f}-1$ partial upper bounding functions. 


$$
F_{1}(x)=\frac{1}{2 M_{1}}\left(\frac{z}{d}+\frac{\mathrm{d} M_{1}}{2}\right)^{2}-\left(\frac{z}{d}-\frac{M_{0} d}{2}\right) x-\frac{M_{0}}{2} x^{2} .
$$

Let $x_{1}$ and $x_{2}$ be the roots of the equation $F_{1}(x)=0$; they are given by

$$
\left\{\begin{aligned}
x_{1}=\frac{1}{M_{0}} & \left\{\left(\frac{M_{0} d}{2}-\frac{z}{d}\right)\right. \\
& \left.-\sqrt{\left(\frac{M_{0} d}{2}-\frac{z}{d}\right)^{2}+\frac{M_{0}}{M_{1}}\left(\frac{M_{1} d}{2}+\frac{z}{d}\right)^{2}}\right\} \\
x_{2}=\frac{1}{M_{0}}\left\{\left(\frac{M_{0} d}{2}-\frac{z}{d}\right)\right. & \\
& \left.+\sqrt{\left(\frac{M_{0} d}{2}-\frac{z}{d}\right)^{2}+\frac{M_{0}}{M_{1}}\left(\frac{M_{1} d}{2}+\frac{z}{d}\right)^{2}}\right\}
\end{aligned}\right.
$$

Then $F_{1}$ is written in the following way

$$
F_{1}(x)=-\frac{M_{0}}{2}\left(x-x_{1}\right)\left(x-x_{2}\right) \text {. }
$$

Let

$$
\begin{gathered}
\alpha=\frac{M_{0} d}{2}+\frac{z}{d}, \\
\theta=\frac{M_{0} d}{2}-\frac{z}{d}, \\
\beta=\sqrt{\left(\frac{M_{0} d}{2}-\frac{z}{d}\right)^{2}+\frac{M_{0}}{M_{1}}\left(\frac{M_{1} d}{2}+\frac{z}{d}\right)^{2}} .
\end{gathered}
$$

We have

$$
\begin{aligned}
\int_{0}^{d} \frac{\mathrm{d} x}{F_{1}(x)} & =\frac{1}{\beta}\left\{\log \left|\frac{\alpha+\beta}{\alpha-\beta}\right|+\log \left|\frac{\theta+\beta}{\theta-\beta}\right|\right\} \\
& =\frac{1}{\beta}\left\{\log \left(\frac{\alpha+\beta}{\beta-\alpha}\right)+\log \left(\frac{\theta+\beta}{\beta-\theta}\right)\right\} .
\end{aligned}
$$

Since $\theta \geq 0$ and $g_{1}$ reaches its minimum at the point $\theta$, then

$$
\frac{\theta+\beta}{\beta-\theta} \geq 1
$$

and

$$
\int_{0}^{d} \frac{\mathrm{d} x}{F_{1}(x)} \geq \frac{1}{\beta} \log \frac{\alpha+\beta}{\beta-\alpha}
$$

Since

$$
\log \frac{\alpha+\beta}{\beta-\alpha} \geq 2 \log \left(1+\sqrt{\frac{M_{0}}{M_{1}+M_{0}}}\right),
$$

and

$$
\frac{1}{\beta} \geq \frac{1}{\sqrt{2\left(M_{1}+M_{0}\right)\left(f^{*}+\varepsilon\right)}},
$$

then

$$
\begin{aligned}
& \int_{0}^{d} \frac{\mathrm{d} x}{f^{*}+\varepsilon-f(x)} \\
\geq & \sqrt{\frac{2}{\left(M_{1}+M_{0}\right)\left(f^{*}+\varepsilon\right)}} \log \left(1+\sqrt{\frac{M_{0}}{M_{1}+M_{0}}}\right),
\end{aligned}
$$

and

$$
\begin{aligned}
& \int_{a}^{b} \frac{\mathrm{d} x}{f^{*}+\varepsilon-f(x)} \\
\geq & n_{r e f} \sqrt{\frac{2}{\left(M_{1}+M_{0}\right)\left(f^{*}+\varepsilon\right)}} \log \left(1+\sqrt{\frac{M_{0}}{M_{1}+M_{0}}}\right) .
\end{aligned}
$$

This proves (6).

Now let us consider the function $G$ defined and continuous on $\left[y_{i}, y_{i+1}\right]$ such that

- $f(x) \leq G(x) \quad \forall x \in\left[y_{i}, y_{i+1}\right]$,

- $G(x) \leq f^{*}$.

Two cases are considered case 1: If

$$
\frac{1}{2 M_{1}}\left(\frac{z}{d}+\frac{M_{1} d}{2}\right)^{2}-\frac{1}{2 M_{0}}\left(\frac{z}{d}+\frac{M_{0} d}{2}\right)^{2} \geq \varepsilon,
$$

then, the function $G$ is given by

$$
G(x)=g_{2}(x)=\left(\frac{z}{d}+\frac{M_{0} d}{2}\right) x-\frac{M_{0}}{2} x^{2} .
$$

Hence

$$
\int_{0}^{d} \frac{\mathrm{d} x}{f^{*}+\varepsilon-f(x)} \leq \int_{o}^{d} \frac{\mathrm{d} x}{f^{*}+\varepsilon-g_{2}(x)} .
$$

We consider

$$
F_{2}(x)=\frac{1}{2 M_{1}}\left(\frac{z}{d}+\frac{M_{1} d}{2}\right)^{2}-\left(\frac{z}{d}+\frac{M_{0} d}{2}\right) x+\frac{M_{0}}{2} x^{2},
$$

its derivative is

$$
\begin{gathered}
F_{2}^{\prime}(x)=-\left(\frac{z}{d}+\frac{M_{0} d}{2}\right)+M_{0} x, \\
F_{2}^{\prime}(x)=0 \Leftrightarrow x^{*}=\frac{1}{M_{0}}\left(\frac{z}{d}+\frac{M_{0} d}{2}\right) .
\end{gathered}
$$

Thus $F_{2}$ is expressed as follows

$$
F_{2}(x)=F_{2}\left(x^{*}\right)+\frac{M_{0}}{2}\left(x-x^{*}\right)^{2},
$$


hence

$$
\begin{gathered}
\int_{0}^{d} \frac{\mathrm{d} x}{F_{2}(x)} \\
=\sqrt{\frac{2}{M_{0} F_{2}\left(x^{*}\right)}}\left\{\arctan \left(\sqrt{\frac{M_{0}}{2 F_{2}\left(x^{*}\right)}}\left(d-x^{*}\right)\right)\right. \\
\left.+\arctan \left(\sqrt{\frac{M_{0}}{2 F_{2}\left(x^{*}\right)}} x^{*}\right)\right\} .
\end{gathered}
$$

Since

$$
F_{2}\left(x^{*}\right)=\frac{1}{2 M_{1}}\left(\frac{M_{1} d}{2}+\frac{z}{d}\right)^{2}-\frac{1}{2 M_{0}}\left(\frac{M_{0} d}{2}+\frac{z}{d}\right)^{2} \geq \varepsilon
$$

and

$$
\arctan x \leq \frac{\pi}{2}
$$

then

$$
\int_{0}^{d} \frac{\mathrm{d} x}{F_{2}(x)} \leq \pi \sqrt{\frac{2}{M_{0} \varepsilon}}
$$

and

$$
\int_{a}^{b} \frac{\mathrm{d} x}{f^{*}+\varepsilon-f(x)} \leq n_{r e f} \pi \sqrt{\frac{2}{M_{0} \varepsilon}} .
$$

Moreover, the inequality

$$
\left(\frac{M_{1}-M_{0}}{2 M_{1}}\right)^{2}\left(f^{*}+\varepsilon\right) \geq \varepsilon
$$

implies (8), this proves the first inequality of (7).

Case 2: Suppose that:

$$
\frac{1}{2 M_{1}}\left(\frac{M_{1} d}{2}+\frac{z}{d}\right)^{2}-\frac{1}{2 M_{0}}\left(\frac{M_{0} d}{2}+\frac{z}{d}\right)^{2} \leq \varepsilon .
$$

Let $X_{1}$ et $X_{2}$ be the roots of the equation

$$
\frac{1}{2 M_{1}}\left(\frac{z}{d}+\frac{M_{1} d}{2}\right)^{2}-\varepsilon-\left(\frac{z}{d}+\frac{M_{0} d}{2}\right) x+\frac{M_{0}}{2} x^{2}=0,
$$

$X_{1}$ and $X_{2}$ are given by

$$
\left\{\begin{aligned}
X_{1}=\frac{1}{M_{0}} & \left\{\left(\frac{z}{d}+\frac{M_{0} d}{2}\right)\right. \\
& -\sqrt{\left.\left(\frac{z}{d}+\frac{M_{0} d}{2}\right)^{2}-\frac{M_{0}}{M_{1}}\left(\frac{z}{d}+\frac{M_{1} d}{2}\right)^{2}+2 M_{0} \varepsilon\right\}} \\
X_{2}=\frac{1}{M_{0}}\left\{\left(\frac{z}{d}+\frac{M_{0} d}{2}\right)\right. & \\
& \left.+\sqrt{\left(\frac{z}{d}+\frac{M_{0} d}{2}\right)^{2}-\frac{M_{0}}{M_{1}}\left(\frac{z}{d}+\frac{M_{1} d}{2}\right)^{2}+2 M_{0} \varepsilon}\right\} .
\end{aligned}\right.
$$

In this case, $G$ is given by

$$
G(x)=g_{3}(x)=\left\{\begin{array}{l}
\left(\frac{z}{d}+\frac{M_{0} d}{2}\right) x-\frac{M_{0}}{2} x^{2} \\
\text { if } x \in\left[0, X_{1}\right] \cup\left[X_{2}, d\right] \\
\frac{1}{2 M_{1}}\left(\frac{z}{d}+\frac{M_{1} d}{2}\right)^{2}-\varepsilon \\
\text { if } x \in\left[X_{1}, X_{2}\right] .
\end{array}\right.
$$

We have

$$
\begin{aligned}
& \int_{0}^{d} \frac{\mathrm{d} x}{f^{*}+\varepsilon-f(x)} \\
\leq & \int_{0}^{d} \frac{\mathrm{d} x}{f^{*}+\varepsilon-g_{3}(x)} \\
= & \int_{0}^{X_{1}} \frac{\mathrm{d} x}{F_{2}\left(x^{*}\right)+\frac{M_{0}}{2}\left(x-x^{*}\right)^{2}} \\
& +\int_{X_{1}}^{X_{2}} \frac{\mathrm{d} x}{\varepsilon}+\int_{X_{2}}^{d} \frac{\mathrm{d} x}{F_{2}\left(x^{*}\right)+\frac{M_{0}}{2}\left(x-x^{*}\right)^{2}} .
\end{aligned}
$$

Moreover, $M_{0}=M_{1}$ implies the condition (9) and we have

$$
\left\{\begin{array}{l}
F_{2}\left(x^{*}\right)=0 \\
X_{1}=\frac{1}{M_{0}}\left\{\left(\frac{z}{d}+\frac{M_{0} d}{2}\right)-\sqrt{2 M_{0} \varepsilon}\right\} \\
X_{2}=\frac{1}{M_{0}}\left\{\left(\frac{z}{d}+\frac{M_{0} d}{2}\right)+\sqrt{2 M_{0} \varepsilon}\right\}
\end{array}\right.
$$

Therefore

$$
\begin{gathered}
\int_{0}^{X_{1}} \frac{\mathrm{d} x}{\frac{M_{0}}{2}\left(x-x^{*}\right)^{2}} £ \sqrt{\frac{2}{M_{0} \varepsilon}}, \\
\int_{X_{1}}^{X_{2}} \frac{\mathrm{d} x}{\varepsilon}=\frac{4}{\sqrt{2 M_{0} \varepsilon}}, \\
\int_{X_{2}}^{d} \frac{\mathrm{d} x}{\frac{M_{0}}{2}\left(x-x^{*}\right)^{2}} \leq \sqrt{\frac{2}{M_{0} \varepsilon}} \\
\int_{0}^{d} \frac{\mathrm{d} x}{f^{*}+\varepsilon-g_{3}(x)} \leq 4 \sqrt{\frac{2}{M_{0} \varepsilon}} .
\end{gathered}
$$

and

Hence

$$
n_{r e f} \geq \frac{\sqrt{M_{0} \varepsilon}}{4 \sqrt{2}} \int_{a}^{b} \frac{\mathrm{d} x}{f^{*}+\varepsilon-f(x)} .
$$


Table 1. Computational results with $\varepsilon=10^{-2}$.

\begin{tabular}{|c|c|c|c|c|c|c|c|c|}
\hline $\begin{array}{l}\text { Function } \\
\text { number }\end{array}$ & Function $f(x)$ & Constant $M$ & Interval $[\mathrm{a}, \mathrm{b}]$ & $\begin{array}{l}\text { Optimal } \\
\text { value } f_{\text {opt }}\end{array}$ & $\begin{array}{l}\text { Optimal } \\
\text { point(s) } x_{\text {opt }}\end{array}$ & $N_{C}$ & $n_{r e f}$ & $\frac{N_{C}}{n_{r e f}}$ \\
\hline \multirow[t]{2}{*}{1} & $(-3 x+1.4) \sin (18 x)$ & 626.4 & {$[0,1]$} & 1.48907 & 0.96609 & 13 & 7 & 1.85 \\
\hline & & & & & -6.7745761 & & & \\
\hline \multirow[t]{2}{*}{2} & $\sum_{k=1}^{5} k \sin ((k+1) x+k)$ & 350 & {$[-10,10]$} & 12.03125 & -0.49139 & 76 & 44 & 1.72 \\
\hline & & & & & 5.791785 & & & \\
\hline 3 & $10 x \sin 10 x$ & 2100 & {$[0,1]$} & 7.9197273 & 0.79785 & 16 & 10 & 1.6 \\
\hline \multirow[t]{2}{*}{4} & $-\sin x-\sin 10 x / 3$ & 12 & {$[2.7,7.5]$} & 1.899599 & 5.145735 & 11 & 6 & 1.83 \\
\hline & & & & & -7.0835 & & & \\
\hline \multirow[t]{2}{*}{5} & $\sum_{k=1}^{5} k \cos ((k+1) x+k)$ & 350 & {$[-10,10]$} & 14.508 & -0.8003 & 69 & 39 & 1.76 \\
\hline & & & & & 5.48286 & & & \\
\hline 6 & $e^{-x} \sin 2 \pi x$ & 51 & {$[0,4]$} & 0.788685 & 0.224885 & 21 & 13 & 1.61 \\
\hline 7 & $\left(16 x^{2}-24 x+5\right) e^{-x}$ & 24 & {$[1.9,3.9]$} & 3.85045 & 2.868 & 16 & 11 & 1.45 \\
\hline \multirow[t]{2}{*}{8} & $25 x-128 x^{2}+282.5 x^{3}$ & 370 & {$[0,1]$} & 1.72866 & 0.18916 & 33 & 24 & 1.37 \\
\hline & $-278.7 x^{4}+100.9 x^{5}$ & & & & & & & \\
\hline 9 & $-\sin x-\sin 2 x / 3$ & 9 & {$[3.1,20.4]$} & 1.90596 & 17.029 & 30 & 19 & 1.57 \\
\hline 10 & $x-\sin 3 x+1$ & 10 & {$[0,6.5]$} & 7.81567 & 5.87287 & 11 & 6 & 1.83 \\
\hline 11 & $x \sin x$ & 31 & {$[0,10]$} & 7.91673 & 7.9787 & 22 & 12 & 1.83 \\
\hline \multirow[t]{2}{*}{12} & $-2 \cos x-\cos 2 x$ & 15 & {$[-1.57,6.28]$} & 1.5 & 2.094 & 26 & 17 & 1.52 \\
\hline & & & & & 4.189 & & & \\
\hline 13 & $-\sin ^{3} x-\cos ^{3} x$ & 18 & {$[0,6.28]$} & 1 & 3.142 & 28 & 18 & 1.55 \\
\hline \multirow[t]{2}{*}{14} & $-\sin x-\sin \frac{10 x}{3}$ & 12.1 & {$[2.7,7.5]$} & 1.6013 & 5.19978 & 11 & 6 & 1.83 \\
\hline & $-\log x+0.84 x-3$ & & & & & & & \\
\hline
\end{tabular}

Table 2. The number of function evaluations of modified Piyavskii's algorithm for different values of $\varepsilon$ and $M$.

\begin{tabular}{|c|c|c|c|c|c|c|c|c|c|c|c|c|c|c|c|}
\hline \multirow[b]{2}{*}{$M \varepsilon$} & \multicolumn{5}{|c|}{ Test function No 1} & \multicolumn{5}{|c|}{ Test function No 2} & \multicolumn{5}{|c|}{ Test function No 3} \\
\hline & 626.4 & 800 & 975 & 1500 & 2000 & 350 & 400 & 500 & 600 & 1000 & 2100 & 2150 & 2200 & 2300 & 3000 \\
\hline $10^{-2}$ & 13 & 17 & 21 & 24 & 26 & 76 & 78 & 85 & 86 & 122 & 16 & 16 & 17 & 17 & 18 \\
\hline $10^{-4}$ & 17 & 22 & 25 & 28 & 32 & 88 & 89 & 95 & 103 & 141 & 22 & 22 & 22 & 22 & 23 \\
\hline $10^{-6}$ & 20 & 26 & 31 & 36 & 38 & 96 & 98 & 108 & 118 & 160 & 27 & 27 & 28 & 28 & 29 \\
\hline $10^{-8}$ & 24 & 29 & 36 & 41 & 46 & 106 & 111 & 121 & 132 & 177 & 31 & 32 & 34 & 33 & 36 \\
\hline
\end{tabular}

\section{Computational Experiences}

In this section, we report the results of computational experiences performed on fourteen test functions (see Tables 1 and 2). Most of these functions are test functions drawn from the Lipschitz optimization literature (see
Hansen and Jaumard [20]).

The performance of the Modified Piyavskii's algorithm is measured in terms of $N_{C}$, the number of function evaluations. The number of function evaluations $N_{C}$ is compared with $\left(n_{r e f}\right)$, the number of function evaluations required by the reference sequential algorithm. We ob- 
serve that $N_{C}$ is on the average only 1.35 larger than $\left(n_{r e f}\right)$. More precisely, we have the following estimation

$$
1.35 \leq \frac{N_{C}}{n_{r e f}} \leq 1.85 .
$$

For the first three test functions, we observe that the influence of the parameter $M$ is not very important, since the number of function evaluations increase appreciably for a same precision $\varepsilon$.

\section{REFERENCES}

[1] R. Horst and H. Tuy, "Global Optimization-Deterministic Approaches," 2nd Edition, Springer, Berlin, 1992.

[2] S. A. Piyavskii, "An Algorithm for Finding the Absolute Minimum of a Function," USSR Computational Mathematics and Mathematical Physics, Vol. 12, No. 4, 1967, pp. 13-24.

[3] S. A. Piyavskii, "An Algorithm for Finding the Absolute Minimum of a Function," USSR Computational Mathematics and Mathematical Physics, Vol. 12, No. 4, 1967, pp. 57-67. doi:10.1016/0041-5553(72)90115-2

[4] B. O. Shubert, "A Sequential Method Seeking the Global Maximum of a Function," SIAM Journal on Numerical Analysis, Vol. 9, No. 3, 1972, pp. 379-388. doi: $10.1137 / 0709036$

[5] P. Basso, "Iterative Method for the Localization of the Global Maximum," SIAM Journal on Numerical Analysis, Vol. 19, No. 4, 1982, pp. 781-792. doi:10.1137/0719054

[6] P. Basso, "Optimal Search for the Global Maximum of Function with Bounded Seminorm," SIAM Journal on Numerical Analysis, Vol. 22, No. 5, 1985, pp. 888-903. doi:10.1137/0722053

[7] F. Schoen, "On a Sequential Search Strategy in Global Optimization Problems," Calcolo, Vol. 19, No. 3, 1982, pp. 321-334. doi:10.1007/BF02575808

[8] Z. Shen and Y. Zhu, "An Interval Version of Shubert's Iterative Method for the Localization of the Global Maximum," Computing, Vol. 38, No. 3, 1986, pp. 275280. doi:10.1007/BF02240102

[9] R. Horst and H. Tuy, "On the Convergence of Global Methods in Multi extremal Optimization," Journal of Optimization Theory and Applications, Vol. 54, No. 2, 1987, pp. 253-271. doi:10.1007/BF00939434

[10] Y. D. Sergeyev, "Global One-dimensional Optimization Using Smooth Auxiliary Functions," Mathematical Programming, Vol. 81, No. 1, 1998, pp. 127-146. doi:10.1007/BF01584848

[11] D. R. Jones, C. D. Perttunen and B. E. Stuckman, "Lipschitzian Optimization without the Lipschitz Constant," Journal of Optimization Theory and Applications, Vol. 79, No. 1, 1993, pp. 157-181. doi:10.1007/BF00941892

[12] Y. M. Danilin and S. A. Piyavskii, "An Algorithm for Finding an Absolute Minimum," USSR Computational Mathematics and Mathematical Physics, Vol. 12, No. 4,
1967, pp. 25-37.

[13] D. Q. Mayne and E. Polak, "Outer Approximation Algorithm for Non Differentiable Optimization Problems," Journal of Optimization Theory and Applications, Vol. 42, No. 1, 1984, pp. 19-30. doi:10.1007/BF00934131

[14] R. H. Mladineo, "An Algorithm for Finding the Global Maximum of Multimodal, Multivariate Function," Mathematical Programming, Vol. 34, No. 2, 1986, pp. 188-200. doi:10.1007/BF01580583

[15] C. C. Meewella and D. Q. Mayne, "An Algorithm for Global Optimization of Lipschitz Functions," Journal of Optimization Theory and Applications, Vol. 57, No. 2, 1988, pp. 307-323. doi:10.1007/BF00938542

[16] Y. G. Evtushenko, "Numerical Methods for Finding the Global Extremum of a Function," USSR Computational Mathematical Physics, Vol. 11, No. 6, 1971, pp. 38-54. doi:10.1016/0041-5553(71)90065-6

[17] E. A. Galperin, "The Cubic Algorithm," Journal of Mathematical Analysis and Applications, Vol. 112, No. 2, 1985, pp. 635-640. doi:10.1016/0022-247X(85)90268-9

[18] P. Hansen, B. Jaumard and S. H. Lu, "Global Optimization of Univariate Lipschitz Functions: I. Survey and Properties," Mathematical Programming, Vol. 55, No. 1-3, 1992, pp. 251-272. doi:10.1007/BF01581202

[19] P. Hansen, B. Jaumard and S. H. Lu, "Global Optimization of Univariate Lipschitz Functions: II. New Algorithms and Computational Comparaison," Mathematical Programming, Vol. 55, No. 1-3, 1992, pp. 273-292. doi:10.1007/BF01581203

[20] P. Hansen and B. Jaumard, "Lipschitz Optimization," In: R. Horst and P. M. Pardalos, Eds., Handbook of Global Optimization, Kluwer Academis Publishers, Dordrecht, 1994, pp. 407-493.

[21] R. P. Brent, "Algorithms for Minimization without Derivatives," Prentice-Hall, Englewood Cliffs, 1973.

[22] S. E. Jacobsen and M. Torabi, "A Global Minimization Algorithm for a Class of One-Dimensional Functions," Journal of Mathematical Analysis and Applications, Vol. 62, No. 2, 1987, pp. 310-324. doi:10.1016/0022-247X(78)90128-2

[23] L. Breiman and A. Cutler, "A Deterministic Algorithm for Global Optimization," Mathematical Programming, Vol. 58, No. 1-3, 1993, pp. 179-199. doi:10.1007/BF01581266

[24] W. Baritompa and A. Cutler, "Accelerations for Global Optimization Covering Methods Using Second Derivatives," Journal of Global Optimization, Vol. 4, No. 3, 1994, pp. 329-341. doi:10.1007/BF01098365

[25] Y. M. Danilin, "Estimation of the Efficiency of an absolute Minimum Finding Algorithm," USSR Computational Mathematical Physics, Vol. 11, No. 4, 1971, pp. 261-267. doi:10.1016/0041-5553(71)90020-6

[26] P. Hansen, B. Jaumard and S. H. Lu, "On the Number of Iterations of Piyavskii's Global Optimization Algorithm," Mathematics of Operations Research, Vol. 16, No. 2, 1991, pp. 334-350. doi:10.1287/moor.16.2.334 